Due to the excellent prognosis, some authors affirm that it is possible to perform a conservative surgery in young women who have not fulfilled their reproductive desire, without having repercussions in terms of overall survival.

The objective of the study was to know the treatment and the overall survival of the Borderline Ovarian Tumors in young women.

Methodology A retrospective cohort study was conducted. The study period was from January 2012 to January 2020. A total of 131 women with Borderline Ovarian Tumors were included in the study. Information was collected about: demographic data, diagnosis, type of surgery performed, disease-free survival, and overall survival. Two cohorts were established: conservative treatment $(n=37)$ vs radical treatment $(n=94)$.

Result(s)* 131 women with a diagnosis of Borderline Ovarian Tumors were diagnosed, representing $16.7 \%$ of all the malignant ovarian tumors within this period ( $\mathrm{n}=784$ ). 91.5\% were diagnosed in Stage I according to the FIGO classification and the most histological line frequent was the mucinous (49.2\%). The risk of global relapse was $6.2 \%$ and overall survival was $95.4 \%$.

Regarding the group of patients who underwent conservative surgery, the risk of relapse was $13.5 \%(n=5)$ compared to $3.2 \%(\mathrm{n}=3)$ in the group radical treatment, $(\mathrm{p}=0.042)$.

The Overall survival was $100 \%$ in women with conservative surgery compared to radical surgery, 94.6\%, $(\mathrm{p}=0.130)$. Conclusion* Borderline Ovarian Tumors present excellent prognosis, obtaining great positive results in overall survival regarding the performance of conservative surgery in young women with unfulfilled reproductive desire.

\section{SUCCESSFUL LIVE BIRTH TWINS AFTER CHEMOTHERAPY DUE CARCINOMA OF COLON DURING PREGNANCY'S: A CASE REPORT}

${ }^{1} B$ Jocic Pivac*, 'V Krsic, ${ }^{2}$ J Milojevic. 'GAK Narodni front, obgyn, Belgrade, Serbia; ${ }^{2}$ general hospital Lazarevac, obgyn, Belgrade, Serbia

\subsection{6/ijgc-2021-ESG0.241}

Introduction/Background* Diagnosing cancer during pregnancy is uncommon. Approximately one in every 1000 pregnancies is diagnosed with cancer during the antenatal period. Cervical cancer and breast cancer are among the most commonly identified cancers during pregnancy but gastrointestinal, urological, and lung cancers have a lower rate of incidence.

In this case we discuss successful management of a patient who was diagnosed with carcinoma of ascendant colon during the antenatal period.

Methodology 42 years old women (PARA -0, GRAV -) was admitted in our hospital in 12 weeks gestations because of pain in abdomen. In previous history, before 4 years she had breast cancer surgery when a local tumorectomy was performed with chemotherapy in six cycles. The pregnancy occurred after IVF. She had all symptoms of hyperstimulation syndromes with big ovaries in diameter of $12 \mathrm{~cm}$ and ascites. She did not complain any gastrointestinal problem. After two weeks of therapy for hyperstimulation syndromes she had increasing abdominal pain. We performed laparotomy.

Result(s)* After opening the abdomen, we found a tumor of the ascending colon and performed a right hemicolectomy with bilateral ovariectomy. The omentum was full of metastases. We performed a colostomy. Her abdominal pain was lost after surgery. She received five cycle of hemotherapy of FOLOFOX. She was delivered by Caesarean section at the $33^{\text {rd }}$ weeks of pregnancy. She was discharged on the seven postoperative day because of her good general condition, as well as that of her newborns, two girls.

Conclusion* Carcinoma and pregnancy bring us two main problems. One is to discover right diagnosis and the other is to choose optimal treatment because it requires considerations of both pregnant women and unborn fetus. Colorectal carcinomas are rare and definitive treatment depends on the term of pregnancy. Generally, definitive surgical therapy is offered if the diagnosis is made early in the pregnancy and deferred until after parturition if it made in the late pregnancy. In our case we performed even hemotherapy because of due to the advanced stage of the disease

\section{FERTILITY SPARING TREATMENT IN PATIENTS WITH ENDOMETRIAL CANCER (FERT-ENC) FROM THE SPANISH INVESTIGATIONAL NETWORK GYNECOLOGIC ONCOLOGY GROUP}

${ }^{1} \mathrm{~V}$ Lago*, ${ }^{1} \mathrm{MT}$ Marina Martín, ${ }^{2} \mathrm{M}$ Laseca Modrego, ${ }^{3} \mathrm{~B}$ Gil Ibanez, ${ }^{4} \mathrm{JR}$ Rodriguez, 5f Domingo, ${ }^{6} \mathrm{~L}$ Minig, ${ }^{1} \mathrm{P}$ Padilla Iserte, ${ }^{2} \mathrm{O}$ Arencibia Sanchez, ${ }^{7} \mathrm{M}$ Sala Ferichola, ${ }^{8} \mathrm{M}$ Munmany, ${ }^{9} \mathrm{~B}$ Martin, ${ }^{10 ; 11} \mathrm{~S}$ lacoponi, ${ }^{12} \mathrm{~S}$ Cabrera, ${ }^{13} \mathrm{P}$ Coronado, ${ }^{14} \mathrm{~J}$ Utrilla Layna, ${ }^{15} \mathrm{~A}$ Bataller Calatayud, ${ }^{16} \mathrm{G}$ Fiol, ${ }^{17} \mathrm{~S}$ Corbalán, ${ }^{1} \mathrm{~S}$ Domingo. ${ }^{1}$ University Hospital La Fe, Spain; ${ }^{2}$ Maternal and Child University Hospital of the Canarias; ${ }^{3}$ University Hospital 12 de Octubre; ${ }^{4}$ Clinic and University Hospital Virgen de la Arrixaca; ${ }^{5}$ VII Las Palmas; ${ }^{6}$ CEU Cardenal Herrera University; ${ }^{7}$ General University hospital of Alicante; ${ }^{8}$ Clinic University Hospital; ${ }^{9}$ University Hospital Getafe; ${ }^{10}$ Hospital Quironsalud Madrid; ${ }^{11}$ University Hospital Quironsalud Madrid, Gynecology, Pozuelo de Alarcón, Spain; ${ }^{12}$ University hospital Vall d' hebron; ${ }^{13}$ Clinic University Hospital San Carlos; ${ }^{14}$ University Hospital Fundación Jimenez Díaz; ${ }^{15}$ University Hospital of La Rivera; ${ }^{16}$ University Hospital Torrecárdenas; ${ }^{17}$ University Hospital Los Arcos

\subsection{6/ijgc-2021-ESG0.242}

Introduction/Background* The primary objective was to evaluate the response rate of conservative treatment in endometrial cancer and the secondary objective was to assess oncological, fertility and obstetric outcomes in fertility preservation patients

Methodology This was a multi-centre, observational, retrospective study of endometrial cancer patients who underwent fertility-sparing treatment in Spanish centres (January 20102020). Seventy-three patients with stage IA, endometrioid adenocarcinoma of the uterus were included in the study.

Result(s)* The levonorgestrel intrauterine device (LNG-IUD) was the most used method (53.4\%), followed by megestrol acetate $(20.5 \%)$ and medroxyprogesterone acetate (16.4\%). During the 24-month follow-up, the rate of complete response to the fertility-sparing management was 74\% ( $\mathrm{n}=$ $54 / 73)$ and $8.2 \%(n=6 / 73)$ patients presented a partial response. Additionally, $13(17.8 \%)$ patients presented with persistent disease and six (8.2\%) patients relapsed after response. The use of LNG-IUD was associated with a higher complete response rate (LNG-IUD $87.2 \%$ vs. others $58.8 \%$; $\mathrm{p}=0.01$ ). Surgical treatment (at least hysterectomy) was completed in $44(60.3 \%)$ patients. Four $(5.5 \%)$ patients presented with relapse after surgery being associated with it the final stage FIGO III $(\mathrm{p}=0.036)$, myometrial invasion $>$ 
$50 \%(\mathrm{p}=0.018)$, and final tumour grade $2-3(\mathrm{p}=0.018)$. The mean follow-up was 57.8 (range 6-159) months. The five-year relapse-free survival and overall survival were 92.6\% (95\% CI: 81.3-97.2\%) and 93.5\% (95\% CI: $80.7-$ $97.9 \%)$, respectively. The rate of pregnancy was $81.1 \%(\mathrm{n}=$ $30 / 37$ ), using, in most cases, reproductive techniques $(78.4 \%)$ for this purpose.

Conclusion* Fertility-sparing management presents a high response rate in endometrial cancer. The use of LNG-IUD associates a better response rate, when compared to other treatment options. Moreover, pregnancy can be achieved with this management by use of reproductive techniques.

\section{THE IMPACT OF TUMOR SIZE ON ONCOLOGICAL OUTCOMES OF FERTILITY PRESERVATION SURGERY IN EARLY CERVICAL CANCER: A MULTI-CENTRIC STUDY BY SPAIN- GOG}

${ }^{1} \mathrm{~B}$ Gil Ibanez ${ }^{*},{ }^{2} \mathrm{~A}$ Torne, ${ }^{3} \mathrm{~A}$ Gil-Moreno, ${ }^{4} \mathrm{~A}$ Martin, ${ }^{5} \mathrm{G}$ Mancebo, ${ }^{6} \mathrm{P}$ Coronado, ${ }^{7}$ MT Marina Martín, ${ }^{8} \mathrm{~A}$ Llueca, ${ }^{2} \mathrm{~B}$ Diaz-Feijoo, ${ }^{1} \mathrm{~A}$ Tejerizo. ${ }^{1}$ Gynecological Oncology and Endoscopy Unit. Gynecology and Obstetrics Department. University Hospital 12 de Octubre. Madrid. Spain. Research Institute i+12. University Hospital 12 de Octubre., Spain; ${ }^{2}$ Unitat de Ginecologia Oncologica. ICGON. Endocrinology, Gynecology and Human Reproduction_IDIBAPS. Hospital Clínic de Barcelona., Spain; ${ }^{3} 3$ Gynecological Oncology Department, Hospital Universitari Vall d'Hebron, Universitat Autònoma de Barcelona, Barcelona, Spain. Centro de Investigación Biomédica en Red de Cáncer, CIBERONC, Madrid. , Spain; ${ }^{4}$ University Hospital Son Llàtzer. Mallorca; ${ }^{5}$ Hospital Parc de Salut Mar. Barcelona., Spain; ${ }^{6}$ Hospital Clínico. Madrid, Spain; ${ }^{7}$ Hospital La Fe. Valencia.Spain, Spain; ${ }^{8}$ Gynecology Oncology Unit Hospital General Universitario de Castellon. Departamento de Medicina. Universitat Jaume I (UJ). , Spain

\subsection{6/ijgc-2021-ESGO.243}

Introduction/Background* The combination of improved survival and delaying child bearing increases the trend of early cervical cancer diagnosis in women without a fulfilled gestational wish. Fertility preservation surgery (FSS) becomes a necessary treatment option for young women.

The aim of this study is to analyze the impact of tumor size on surgical and oncological outcomes of FSS in early cervical cancer in Spain.

Methodology A multicenter, retrospective cohort study of early cervical cancer (IA2- IB1, FIGO 2009) patients with gestational desire who underwent FSS was carried out at the departments of gynecology of 12 tertiary care hospitals between 01/2005 and 01/2019 throughout Spain. The data were registered in an on-line database. All analyses were performed using STATA 15 statistical software

Result(s)* A total of 111 patients who underwent trachelectomy were included, $82(73.9 \%)$ of them with tumors $<2$ $\mathrm{cm}$ and $29(26.1 \%)$ of them $\geq 2 \mathrm{~cm}$. Patients' characteristics were balanced except linfovascular space infiltration (LVSI). All patients were intraoperative node negative. There were no significant differences between groups regarding surgical approach, performance of a posterior cerclage, intraoperative complications or need of posterior hysterectomy.

Median follow-up was 55.7 months in patients group $<2$ $\mathrm{cm}$ tumor and 30.7 months in group $\geq 2 \mathrm{~cm}$. Eleven recurrences were diagnosed $(9.9 \%), 5(6.0 \%)$ in the $<2 \mathrm{cms}$ tumor group and $6(21.4 \%)$ in the $\geq 2 \mathrm{cms}$ tumor group $(\mathrm{p}<0.05)$.

Cox regression was performed to identify different predictor factor for recurrence. Only tumor size $(<2 \mathrm{~cm}$ vs. $\geq 2 \mathrm{cms})$ was found to be significant among histology, LVSI, previous conization or surgical approach. After adjusting for the rest of the variables, tumor size $\geq 2 \mathrm{~cm}$ has a Hazard Ratio of 5.99 (CI 95\% 1.01-35.41, $\mathrm{p}=0.036$ )

Conclusion* This study shows a real world data of a large number of trachelectomies performed in patients with early cervical cancer after negative lymphnode assessment. Selection criteria should be rigorous especially for patients with tumor $\geq 2 \mathrm{~cm}$ due to its worse oncological outcomes. Options may be discussed with the patient to reach a balance between the risk of recurrence and the best fertility results

\section{THE ADVISORY BOARD ON CANCER, INFERTILITY AND PREGNANCY}

1;2J Heimovaara, ${ }^{3 ; 4} \mathrm{~K}$ Van Calsteren, ${ }^{2} \mathrm{C}$ Lok, ${ }^{1 ; 2} \mathrm{~F}$ Amant* ${ }^{*}$ Katholieke Universiteit Leuven, Department of Oncology, Leuven, Belgium; ${ }^{2}$ The Netherlands Cancer Institute (NKI), Department of Gynaecology, Amsterdam, Netherlands; ${ }^{3}$ University Hospitals Leuven, Department of Obstetrics and Gynecology, Leuven, Belgium; ${ }^{4}$ Katholieke Universiteit Leuven, Department of Development and Regeneration, Leuven, Belgium

\subsection{6/ijgc-2021-ESGO.244}

Introduction/Background* Due to the rarity of cancer during pregnancy, physicians not always have up to date knowledge on all possibilities of treatment during pregnancy. To support physicians with decision on optimal cancer treatment for their pregnant patients, the Advisory Board on Cancer, Infertility and Pregnancy (ABCIP) was founded under the umbrella of the International Network for Cancer, Infertility and Pregnancy (INCIP).

Methodology Medical experts from a variety of disciplines and countries were informed on this new initiative, and asked whether they were interested in actively participating in the ABCIP. Several national advisory boards were founded, as well as an overarching international board. Funding was acquired to build a website to facilitate accessibility for physicians requesting advice and a forum for online discussions and communication between the board members. Based on available literature, default texts were drafted to use in the recommendation letters.

Result(s)* An online platform was created at www.ab-cip.org, where physicians are able to submit questions regarding their pregnant patients with cancer, using only anonymous patient data. A total of 73 experts from a variety of disciplines and countries are joined in the national and international boards. Of this group, experts from eight countries already expressed their interest in setting up their own national advisory board. Incoming requests are discussed by the experts on a secure forum on the website. The website generates a recommendation letter, based on available literature and expert opinion of the board members, to send back to the requesting physician. In the first month after foundation, seven requests for advice were already submitted and discussed.

Conclusion* The ABCIP provides easily accessible, free of charge recommendations to physicians from all over the world with questions regarding their pregnant patients with a cancer diagnosis or patients with a cancer diagnosis who wish to become pregnant in the future. 